Mots. Les langages du politique

$72 \mid 2003$

La ville, entre dire et faire

\title{
Le tout et la partie : des catégories de l'urbain à
} nommer

The entirety and the part: naming urban categories

El todo y la parte : nombrar las categorias de lo urbano

\section{Olivier Ratouis}

\section{CpenEdition}

Journals

Édition électronique

URL : https://journals.openedition.org/mots/5433

DOI : $10.4000 /$ mots.5433

ISSN : 1960-6001

Éditeur

ENS Éditions

Édition imprimée

Date de publication : 1 juillet 2003

Pagination : 13-27

ISBN : 2-84788-034-8

ISSN : 0243-6450

\section{Référence électronique}

Olivier Ratouis, «Le tout et la partie : des catégories de l'urbain à nommer », Mots. Les langages du politique [En ligne], 72 | 2003, mis en ligne le 28 avril 2008, consulté le 24 avril 2022. URL : http:// journals.openedition.org/mots/5433; DOI : https://doi.org/10.4000/mots.5433

\section{(c) ENS Éditions}


Olivier RATOUIS ${ }^{\circ}$

\section{Le tout et la partie: des catégories de l'urbain à nommer}

De la reconstruction d'après-guerre au projet urbain de la fin du siècle, la production de l'urbain s'accompagne de mots divers pour la qualifier $^{\circ \circ}$. La seule particularité des phases successives de projets et d'opérations est-elle suffisante pour expliquer les évolutions de sens et les changements de mots? À Dunkerque, de la ville nouvelle, en même temps ville de toujours, à reconstruire dans les années 40 , au projet de «faire de l'agglomération une vraie ville» dans les années 90, en passant par la grande expansion urbaine des années 60 , les contextes sont effectivement très différents. Repérer ces permanences et ces changements à travers ces noms offre une manière renouvelée de poser la question urbaine. Ne faudrait-il pas nommer la ville pour la faire?

Deux catégories de l'urbain nous intéressent ici particulièrement, celle du tout (ville, agglomération...) et celle de la partie (zone, quartier...). L'analyse du vocabulaire des textes de présentation et d'accompagnement des projets (issus des différents fonds d'archives) ${ }^{1}$ permet de contribuer à la définition des phases, à l'identification des ruptures dans la

- Historien et urbaniste, Institut des mers du Nord, Université du Littoral-Côte d'Opale, 21, quai de la citadelle, BP 5528, Dunkerque cedex 1, oratouis@club-internet.fr.

○ Mots étudiés utilisés par les acteurs: agglomération; grand ensemble; projet urbain; quartier; reconstruction; ville (ville nouvelle, ville de toujours, vraie ville, grande ville); zone; ZUP.

1. Étudiés à l'occasion de plusieurs recherches antérieures, en particulier: O. Ratouis, 1997, Dunkerque ou la question de la ville comme totalité, de la reconstruction aux années soixante-dix, Doctorat, Paris, EHESS, 657 p., et O. Ratouis, M. Segaud, 2000, Vers une maitrise d'ouvrage territoriale? Les projets urbains du littoral Nord-Pas-de-Calais entre aménagement et développement, Plan urbain construction et architecture, Institut des mers du Nord, 167 p. 
grande transformation urbaine de la seconde moitié du $20^{\mathrm{e}}$ siècle. On relève dans l'après-guerre, à contre-courant du colportage organisé des discours volontaires et annonciateurs de mondes nouveaux, des doutes, et même un flou sémantique, par exemple chez les principaux responsables de l'administration chargés de mettre en œuvre ces politiques. Différemment, lorsque la crise se manifeste plus durement à partir des années 70 , l'invention reprend et le discours aide à faire corps et produire, à côté de discours particuliers, des énoncés plus collectifs — permettant par exemple de proposer le concept de "collectif d'énonciation» des projets d'aménagement ${ }^{2}$. Peut-on alors corréler des types d'énoncés et des types de production urbaine?

\section{Faire de l'agglomération une ville?}

Deux exemples permettent d'illustrer l'intérêt d'une approche historique de temps long, c'est-à-dire ici, dont la durée est supérieure à celle des opérations. Comment parle-t-on du projet? En décembre 1957, l'urbaniste Gérard Deldique est chargé de préparer l'arrivée de la sidérurgie à Dunkerque (Usinor); il fixe comme objectif principal au plan directeur d'aménagement de «faire de l'agglomération dunkerquoise une ville» ${ }^{3}$. En 1991, le Contrat d'agglomération, qui organise les projets territoriaux des années 90, a pour mot d'ordre le renforcement de l'intercommunalité dans l'objectif de "faire de l'agglomération une vraie ville» ${ }^{4}$. En mai 1942 , en vue de la reconstruction de la ville détruite par les bombardements de 1940, les urbanistes Roger Puget et Jean Canaux entendent «faire de Dunkerque une vaste agglomération auprès d'un grand port» 5 .

2. Dans les recherches précitées, le concept de «collectif d'énonciation» désigne certaines formes d'ateliers d'urbanisme qui donnent voix à l'ensemble des acteurs intéressés. Voir également J.-Y. Toussaint, 1995, «Le collectif d'énonciation de l'espace ou l'histoire des acteurs que cachait l'architecte», Cahiers $d u$ LAUA, ${ }^{\circ} 3$, École d'architecture de Nantes: «Le projet pourrait se définir comme une institution qui aurait pour fonction, au sein des sociétés contemporaines, la transformation et l'administration de leur territoire. Le Plan serait donc un énoncé collectif, résultat de l'activité productive d'un groupe énonciateur d'espace» (p. 17).

3. G. Deldique, 1957, Groupement régional d'urbanisme de Dunkerque. Premières propositions d'aménagement, $45 \mathrm{p}$.

4. CUD, 1991, Contrat d'agglomération de Dunkerque, p. 22.

5. Archives nationales (AN), Archives du ministère de la Reconstruction et de l'Urbanisme (AMRU), AFU 10129. 
En 1974, le nouveau Schéma directeur d'aménagement et d'urbanisme (SDAU) se donne pour ambition de «réaliser la grande ville auprès du grand port». Dans les années 20, pour la réalisation du Projet d'aménagement, d'extension et d'embellissement (PAEE) de la ville, l'architecte Agache se donnait déjà le même slogan. Constituée à cette occasion d'ailleurs, une Entente intercommunale, rassemblant les huit communes de l'agglomération dunkerquoise, affirme la nécessité d'établir un unique plan d'ensemble et non pas différents plans communaux ${ }^{6}$. De son côté, Georges Bechmann, qui intervient sur la partie du PAEE portant sur les réseaux urbains, prend explicitement position en faveur du terme d'agglomération (dunkerquoise), préféré à celui de Grand Dunkerque ${ }^{7}$. Que peut-on retirer de ces exemples?

Premièrement, c'est la singularité des formules qui retient l'attention, et leur récurrence: «faire de l'agglomération une ville»; faire «la grande ville auprès du grand port». Elle étonne, car s'il s'agit bien de la même ville, Dunkerque, les contextes diffèrent grandement. Dans un cas, il s'agit d'une politique d'État conduite dans le cadre de l'aménagement du territoire des années 60 , pour laquelle le territoire est avant tout un site d'accueil; dans l'autre, de la définition d'une politique locale résultant de la décentralisation des années 90 , qui se veut soucieuse du territoire et de ses spécificités. Durant ces quelques décennies, la population double presque (d'environ 100000 à 200000 habitants), ce qui montre l'ampleur des transformations que connait la ville. Deuxièmement, le sens de ces formules pose question. Que veut dire «faire de l'agglomération une ville»? La différence parait qualitative. Et qu'est-ce que cela signifie étant donné cette variation des contextes, à la fois quantitatifs (de population) et morphologiques (quartiers entiers nouveaux, fort étalement du bâti)?

Si dans les années 20, Dunkerque est enfermée dans les fortifications, on doit comprendre que ces termes de ville et d'agglomération renvoient à la question des limites urbaines, à leur perpétuelle mouvance aux $19^{\mathrm{e}}$ et $20^{\mathrm{e}}$ siècles : Agache pose comme préalable à son $P A E E$ l'arasement de ces fortifications. C'est d'ailleurs en ce sens que l'on commence à utiliser ce terme d'agglomération, comme la somme de la ville et de sa banlieue - proposition faite notamment par le statisticien Paul Meuriot au tour-

6. Ville de Dunkerque, Délibérations du conseil municipal, 29 mars 1920.

7. D.-A. Agache, G. Bechmann, 1923, Ville de Dunkerque et communes voisines, projet d'aménagement, d'embellissement et d'extension, Paris, Léon Eyrolles, p. 42-58. 
nant du siècle ${ }^{8}$. Ces deux mots sont donc des catégories urbaines du tout, et posent le problème de la découpe spatiale, de la démarcation territoriale. Les utiliser, c'est du même coup souligner les ruptures. Comment les acteurs de la production urbaine pensent-ils les ensembles? Comment relèvent-ils des limites, marquent-ils des différences? Si pour les acteurs des années 90 le tout se saisit à travers la catégorie d'agglomération, la reconstruction tend à identifier la ville avec son centre. Comment faire de l'ensemble une unité, et réciproquement? L'analyse repose sur quatre opérations successives.

\section{La Reconstruction: une ville nouvelle?}

Le terme de Reconstruction est resté pour désigner rétrospectivement deux choses: une période, les années d'après-guerre, et un espace, le centre ville reconstruit. Pourtant, les années 40 et 50 ont connu un débat virulent qui s'est exprimé par une alternative: re-construire ou construire. Le ministre de la Reconstruction, Eugène Claudius-Petit, explique d'ailleurs après coup qu'il aurait souhaité que l'on construise et non pas que l'on re-construise.

À Dunkerque, la portée de la reconstruction se trouve exprimée dans le mot d'ordre de l'urbaniste en charge du Plan de reconstruction et d'aménagement (PRA), Théo Leveau: «Retrouver l'âme de la Cité de Jean Bart à travers une modernisation hardie $»{ }^{9}$. Les élus municipaux le traduisent à leur façon lorsqu'ils affirment que «les propriétaires dunkerquois sont pieds et poings liés entre les mains des architectes du MRU» [ministère de la Reconstruction et de l'Urbanisme ${ }^{10}$, désignant non seulement la contrainte des procédures mais aussi l'apparition d'un être urbain qui leur échappe.

Pourtant, la procédure du remembrement qui consiste à évaluer les biens des sinistrés met à mal l'idée de la table rase dont les villes détruites auraient été les victimes non seulement en raison de la guerre mais surtout de la part d'urbanistes modernes. Car si les bombardements aveugles

8. P. Meuriot, 1897, Des agglomérations urbaines dans l'Europe contemporaine. Essai sur les causes, les conditions, les conséquences de leur développement, Thèse pour le doctorat, Paris, Belin, $475 \mathrm{p}$.

9. Comité de coordination de la reconstruction de Dunkerque du 11 juin 1945. AN, AMRU, AFU 10129.

10. Délibérations du Conseil municipal, 15 avril 1949. 
ont rasé des quartiers d'habitation entiers et, dans le cas de Dunkerque, tout le centre ville, le tribut de la ville reconstruite à l'égard de la ville ancienne est manifeste. Le premier travail de l'urbaniste consistant à évaluer les destructions et à en dresser un plan, les géomètres interviennent pour reconstituer le tracé de l'ancien parcellaire. La ville ancienne sert donc bien de fond de plan à la ville reconstruite qui, comme le disent les textes officiels, n'est «pas la création d'une ville nouvelle». Au centre des débats, le foncier fait l'objet d'une attention remarquable, les instructions du ministère expliquant que «la parcelle ancienne est évaluée [...] en supposant que la Ville soit restée dans l'état où elle se trouvait avant les destructions $\gg{ }^{11}$. Cette supposition vient qualifier toute la reconstruction. L'ambigüité est perceptible: le statut de la ville en projet ne se traduit dans aucun autre mot que celui de reconstruction, que, d'une certaine façon, on ne sait pas définir autrement qu'en parlant du «nouveau visage» du «Dunkerque de toujours».

Le vocabulaire de la résurrection est alors utilisé, voire substitué («Plus qu'une reconstruction, une résurrection, celle de Grande Synthe», commune voisine, titre la Voix du Nord le 27 février 1952). Les mots ayant un préfixe en $r e$-renvoient cependant non pas à l'idée d'une reprise par les urbanistes des espaces de la ville ancienne (des formes de ses rues par exemple), comme ce sera le cas dans les années 90, mais à l'idée d'une ville qui se ré-génère par elle-même. Le vocabulaire en re- est ainsi une variante du vocabulaire lié à une conception organiciste de la ville. Toute référence au vivant ne vient-elle pas conforter cette conception? L'architecte Niermans explique que la reconstruction consiste avant tout à «donner à ses habitants une possibilité de vivre sainement et d'y élever ses enfants dans l'air pur, la lumière et le soleil $»^{12}$; et même, d'engager la «renaissance [de la ville] qui a souffert dans ses œuvres vives et qui doit renaitre dans une nouvelle conception de vie ${ }^{13}$. Une telle répétition de vocabulaire gagne au passage une dimension largement incantatoire.

L'usage du mot ville prend un sens particulier dans des oppositions à l'air ou à l'espace: «La ville fermée, repliée sur elle-même [...] et sans soleil est périmée. Il faut aux habitants et aux nouvelles générations les

11. MRU, mars 1947, Instructions générales sur le remembrement urbain des communes sinistrées.

12. J. Niermans, s.d., Dunkerque, MRU, AM, fN, AC.

13. J. Niermans, s.d., Construction d'immeubles collectifs d'État ISAI, MRU, AM, MRU, ISAI. 
grands espaces $»^{14}$. Bied, le représentant local du MRU, en vient d'ailleurs à proposer un énoncé contradictoire: «La vie sera meilleure, dans des villes complètement sinistrées comme Dunkerque, avec des logements plus sains et mieux aérés $\rangle^{15}$. N'est-ce pas en fait le concept de ville qui se dissout ici, opposé en particulier à celui d'espace?

D'un autre côté, cette réflexion amène à valoriser l'importance du centre. Leveau conçoit deux axes principaux. Le premier qui part de la gare pour rejoindre l'hôtel de ville au nord constitue «la grande artère centrale de la ville». L'axe est-ouest devient «la transversale essentielle du Nouveau Dunkerque ${ }^{16}$ désormais bornée par deux nouvelles places. Le principe de la croisée centrale vient d'être concrétisé. La place Jean Bart «au centre de la ville avec sa statue légendaire constitue le pivot de l'agglomération vers lequel tout semble converger $\rangle^{17}$. Le rôle assigné au centre apparait paradoxal. D'une part, la nouvelle organisation territoriale réserve au centre son ancienne fonction commerciale et semble l'y réduire. D'autre part, le centre se démultiplie: au nord de la place Jean Bart, Leveau situe ce qu'il appelle le centre civique; à l'ouest, sur la Citadelle, le centre administratif; à l'est, autour de la place du Théâtre, le centre culturel.

Deux systèmes cohabitent et s'affrontent. Au pivot de l'agglomération correspond une fonction de carrefour des axes; au centre ville une organisation radio-concentrique. "Le principe du plan», explique le ministère, "a voulu entourer l'agglomération principale actuelle d'une ceinture de jardins publics ${ }^{18}$. Cette idée de ceinture est une réponse à la question qui, pour Leveau, est «un des problèmes essentiels de la cité future : celui de la marge de contact entre la ville et la nature ${ }^{19}$. Or, précisément, l'agglomération, que l'INSEE retient en 1954 comme catégorie statistique, repose sur le principe de la continuité bâtie, autrement dit de la séparation entre la ville et la campagne.

14. J. Niermans, Papiers, AM, fN, divers.

15. Commission de coordination, 23 octobre 1952, AM, ASR.

16. Th. Leveau, 1949, «Reconstruction de Dunkerque», in Urbanisme, $\mathrm{n}^{\circ}$ 117-118, Paris, p. 240.

17. Th. Leveau, mars 1947, Plan de reconstruction et d'aménagement de Dunkerque.

18. Série de courriers entre les administrations de mars 1954 à mai 1955, AN, AMRU, AFU 10129.

19. Th. Leveau, 1965, "Les espaces verts dans la cité future», Revue d'hygiène et de médecine sociale, Paris, Masson, t. 13, n 5, p. 416. 


\section{Les ZUP: villes nouvelles ou zones d'habitation? Des dénominations multiples et fluctuantes}

Au début des années 60, plusieurs analystes du fait urbain s'interrogent sur la validité du vocabulaire urbanistique disponible. En 1961, c'est le directeur de l'Aménagement du territoire au ministère de la Construction, Pierre Randet, l'une des figures les plus éminentes de l'administration française de l'urbanisme, qui juge que le vocabulaire n'est plus en mesure de décrire les transformations en cours. À la notion périmée de ville, Randet préfère celle de magma urbain ${ }^{20}$.

Tandis que dans les années 40 avec le Plan de reconstruction et d'aménagement, l'urbaniste postule l'unité de la ville, aidé par la procédure administrative qui s'attache à la reconstruction des villes, avec les plans d'aménagement et les plans d'urbanisme des années 60, le raisonnement découpe l'ensemble urbain en zones d'habitation et en quartiers. L'unité porte sur la partie et non plus sur le tout. Le lexique tente de suppléer à cette partition mais il la souligne dans le même temps. Avec la création des Zones à urbaniser en priorité (ZUP) le 31 décembre 1958, les professionnels mentionnent désormais des plans de ZUP comme on disait auparavant des plans de villes.

L'urbain se substitue à la ville, le non quantifiable au quantifiable. Mais l'administration perçoit-elle l'ampleur du phénomène d'urbanisation en cours? Des personnalités telles que Jean-François Gravier ne prédisent-elles pas en France «un déclin des villes surpeuplées » ${ }^{21}$ ? La notion de ville ne disparait pas complètement mais elle s'adjoint souvent le qualificatif de nouvelle, sans que l'expression soit encore sédimentée. En 1969 d'ailleurs, le géographe Pierre Merlin constate encore l'ambigüité («la confusion») de la notion de ville nouvelle, dont il propose de fixer l'usage à partir d'une double caractéristique: la mixité urbaine et la création d'un centre ville autonome, "ce qui exclut non seulement les grands ensembles, mais aussi les quartiers nouveaux conçus pour faciliter la croissance d'une agglomération». C'est seulement par la suite que l'usage différencie nettement grands ensembles et

20. Cité dans V. Claude, juin 1994, Les fonctions «études» et l'administration de l'urbanisme. Fonction centrale contre fonction diffuse, MELT, PCA, p. 1.

21. J.-F. Gravier, 1955, «L'industrie et les communautés humaines», automne 1954, dans Centre économique et social de perfectionnement des cadres, Conférences 19521955, CGC, p. 18. 
villes nouvelles. La confusion des notions et l'embarras des acteurs à l'égard des principaux concepts urbanistiques transparaissent clairement dans l'analyse de leurs discours et particulièrement de l'ensemble des documents techniques.

Le terme ville est entièrement effacé des discours administratifs dunkerquois des années 60. Une rare occurrence illustre les difficultés: Albert Denvers, l'un des principaux acteurs de cette opération, évoque ainsi les réalisations de «la ZUP de Grande Synthe ville nouvelle» ${ }^{22}$. Les documents témoignent par contre de l'usage de nombreuses autres expressions. Opération d'urbanisme, ZUP, zone d'habitation, agglomération, opération d'ensemble, zone opération, zone résidentielle, cité, noyau de vie, quartiers d'habitation, sont les plus fréquentes, employées indifféremment dans la désignation des mêmes opérations. Par ailleurs, les acteurs recourent volontiers au terme d'unité, parlant d'unité sidérurgique, d'unité(s) d'habitation, d'unité(s) autonome(s) (d'habitation), d'unité(s) résidentielle(s), et même d'unité d'architecture et d'urbanisme de la zone.

Cette dynamique du lexique est signifiante. Le dispositif de financement des opérations induit une pratique qui substitue structurellement la production urbaine à la production de la ville. La notion de ville n'est pourtant pas totalement absente des projets. Mais seuls y font référence les deux architectes en chef de la ZUP et du Plan directeur d'aménagement du Groupement régional d'urbanisme de Dunkerque (GRUD), Toumaniantz et Deldique, et cela uniquement dans les différents textes de présentation du plan. Par comparaison avec l'ensemble des autres documents administratifs, l'emploi du terme ville prend donc une importance qu'il faut pourtant réduire aussitôt: son usage va décroissant avec l'évolution des projets et cela dans des documents d'urbanisme qui datent euxmêmes de la fin des années 50, c'est-à-dire de la période précédant l'approbation du Plan d'urbanisme de détail de Dunkerque en avril 1962. Autrement dit, lorsque les premiers bâtiments sont livrés, le terme n'apparait déjà plus.

Rarement, l'urbaniste reprend le ton des reconstructeurs en invitant à «donner cohésion et âme». La ville a alors un "visage propre», une «physionomie» mais aussi un «être» et une «vie». Cette approche s'accommode mal de ce qui se met alors en place au ministère de la Recons-

22. Courrier d'A. Denvers à Y. Toumaniantz du 22 septembre 1964, IFA, fT, 435. 
truction. G. Deldique adjure cependant de «ne plus faire de zones d'habitation mais des villes $»^{23} \ldots$

Dès juin 1959, avec les Éléments d'enquête et propositions d'aménagement, le ton est très différent. Toumaniantz et Deldique interviennent dans un cadre qui s'est fortement précisé, la localisation de l'unité sidérurgique et des zones d'habitation étant désormais tranchée. Les architectes préparent alors l'aménagement des zones d'habitation. Ils proposent curieusement de concevoir la ZUP des Nouvelles Synthes comme «la création intégrale d'une ville nouvelle du style grand ensemble ${ }^{24}$. En fait, les desideratas de l'industrie obligent les architectes à adapter le plan au coup par coup. La référence à la ville se raréfie d'elle-même. Lorsqu'en juin 1960 les urbanistes préconisent de «ne plus faire de zones d'habitation", ce n'est plus comme en 1957 pour inviter à faire des villes mais pour faire des cités ${ }^{25}$. Ce glissement sémantique trahit un constat d'échec: l'objet n'est plus celui d'une entité territoriale harmonieusement organisée mais celui d'une entité socio-politique réaffirmée. Or cette dernière ne relève qu'après coup du champ d'action des urbanistes.

\section{Le SDAU de 1974: «réunifier l'agglomération»}

Le Schéma directeur d'aménagement et d'urbanisme du littoral Nord $(S D A U)$ a au contraire pour objectif de repartir de l'urbanisme ${ }^{26}$. Après la loi d'orientation foncière de 1967, les acteurs locaux décident de créer une communauté urbaine, qui regroupe les différentes communes de l'agglomération. La compétence d'urbanisme, comprise par le nouveau président Albert Denvers comme le «levier de l'économie», est prise en main par les acteurs locaux et transférée des communes à la nouvelle structure intercommunale.

À lire le $S D A U$, et différents documents préparatoires, il semblerait que deux schémas y soient présents et s'y enchâssent: l'un, fonctionnel (par zones); l'autre hiérarchisé, par espaces différenciés (centre, agglo-

23. G. Deldique, 1957, GRUD. Premières propositions d'aménagement, p. 30.

24. Y. Toumaniantz, G. Deldique, 1959, GRUD. Dunkerque Ouest. Création d'une zone industrielle et d'une zone d'habitation, éléments d'enquête et propositions d'aménagement, p. 5.

25. G. Deldique, 1960, GRUD, Avant-projet de plan directeur d'aménagement, p. 13.

26. Agur, 1974, Schéma directeur d'aménagement et d'urbanisme du littoral Nord. 
mération...). Le Préambule, qui s'intitule Pour une prévision de l'aménagement de l'espace, note ainsi : «Pour l'habitat [...] un développement hiérarchisé, qui s'appuie en priorité sur l'agglomération existante, dont le centre sera conforté, et qui permettra une croissance progressive des bourgs et des villages ». Un changement de vocabulaire est perceptible, avec l'usage développé du mot quartier: «D'autre part, les quartiers ne seront pas construits au plus près des lieux de travail [c'est-à-dire de l'industrie]» (par opposition à «l'agglomération morcelée dominée par des cités dortoirs proches des lieux de travail $»^{27}$ ).

C'est dans le sens de cette hiérarchie qu'apparait le mot d'ordre de cette nouvelle phase d'aménagement. "Dunkerque, une ville au service de l'homme, [connait] un [...] décalage croissant entre la ville et des industries de dimension internationale commandées d'ailleurs, accroissement désordonné parce que trop rapide» (p. 7). La volonté affirmée de construire «la grande ville auprès du grand port» constitue donc une autre formulation d'un thème dominant à multiples déclinaisons. Le discours argumente ainsi une critique en règle des aménagements décidés par l'État dans les années 60, industriels et urbains. C'est en ce sens que le document fondateur de l'urbanisme dunkerquois des années 70 lance une charge contre la philosophie des zones d'aménagement — résultat de cette politique d'État.

Le Schéma directeur souligne ainsi l'importance quantitative et qualitative des coupures spatiales et mobilise le champ sémantique de la couture. C'est par lui que le vocabulaire de l'agglomération est introduit: "L'éclatement du tissu urbain: L'agglomération actuelle est très morcelée par les canaux et les voies ferrées» (p. 10). Le Schéma directeur se fixe pour objectif de chercher l'unité urbaine, et même de la re-trouver puisqu'elle a été effacée par ces dynamiques de morcellement, renvoyant implicitement à un être mythique, l'agglomération d'avant les coupures, effet appuyé par les nombreuses cartes anciennes de la ville enfermée et unie dans ses fortifications. "Réunifier l'agglomération. Au-delà de la simple continuité physique, l'unité de l'agglomération implique de bonnes liaisons entre quartiers périphériques et centre ville». L'agglomération n'est donc pas seulement un concept. Ce mot fait partie des noms donnés à ce qui fait ensemble: «On tendra à ce que la totalité du littoral dunkerquois devienne un

27. Agur, 1971, Dunkerque 2000. Livre blanc, p. 52. 
seul marché de l'emploi, une seule zone d'équipement, un seul marché du logement [qui devra] contribuer à assumer la croissance de l'ensemble» (p. 13).

Cette opposition entre unité et morcellement n'est pas une originalité. Cependant, le choix des termes est signifiant et cela d'autant plus qu'il prend la forme d'une opposition. On retrouve la même chez l'ingénieur fouriériste Perreymond comme clé d'analyse des problèmes de l'espace parisien avant les transformations d'Haussmann, Fourier ayant lui-même construit cette opposition ${ }^{28}$. Confirmation de la sélection d'un vocabulaire dans un document d'urbanisme à la tonalité progressiste, et même utopiste, affichée.

En opposition à l'ensemble viennent les morceaux; au tout, les parties. Le zonage construit cette deuxième catégorie. Toutefois, l'usage de ce mot reste encore ouvert, la critique plus frontale venant quelques années plus tard. Le Schéma directeur défend une politique de «zones vertes» (p. 37) ou vise «la restructuration générale de la zone urbaine centrale» (p. 67). Dans les années 90, le Projet urbain préfère parler «d'aire urbaine centrale $»^{29}$, ayant fait son deuil de ce zonage. Mais dans les années 70 , la procédure des zones d'aménagement concerté (ZAC) succède à celle des ZUP. "ZUP d'usine-ZAC d'agglomération» tentera en une formule et après coup l'aménageur ${ }^{30}$. Mais le zonage, même réformé, est poursuivi. C'est ainsi que l'opération d'aménagement la plus importante alors est celle de la ZAC du Courghain. Entre l'ancienne ZUP de Grande Synthe et la nouvelle ZAC toute proche, les aménageurs prévoient une «zone tampon ${ }^{31}$ qui doit s'articuler avec «une zone de défense verte $»^{32} \ldots$ Et la nouvelle ZAC n'a-t-elle pas ellemême une «zone centrale $»^{33}$ ? Le champ sémantique s'étoffe d'ailleurs avec la mobilisation d'une autre notion, quand il est prévu en outre une «division de la zone en secteurs $»^{34}$.

28. Voir Frédéric Moret, 2001, «Penser la ville en fouriériste: les projets pour Paris de Perreymond», dans Karen Bowie (coord.), La modernité avant Haussmann. Formes de l'espace urbain à Paris (1801-1853), Paris, Parenthèses, p. 98 et suivantes.

29. Agur, 1994, Projet urbain d'agglomération - aire urbaine centrale.

30. SAEN, 1983, 1960-1980: ZUP d'usine - ZAC d'agglomération.

31. SEDN, Compte rendu de la réunion du 8 juin 1972. Archives municipales de Grande Synthe.

32. Procès-verbal du Conseil communautaire du 29 janvier 1973.

33. Mairie de Grande Synthe, Compte rendu de la réunion publique du 16 janvier 1973.

34. CUD, 1974, Dossier de réalisation de la ZAC du Courghain. 
À Dunkerque, l'agglomération revient en force dès le SDAU de 1974. La notion est convoquée pour décrire et nommer le tout; elle sert également à rassembler, à faire l'union, parmi les différentes communes face à l'État et la grande industrie. Son usage accompagne un changement de locuteur. Le conflit de projets avec l'État est aussi un conflit de discours. La nouvelle agence d'urbanisme (Agur) rédactrice du $S D A U$ fait office de porte-voix du local et choisit ses mots.

\section{Le Projet urbain: l'agglomération comme solution évidente}

La mise en valeur de l'agglomération signe un retournement historique: celui de l'intercommunalité comme plus-value. C'est ainsi un Contrat d'agglomération, et non un Contrat de ville comme ailleurs en France, qui est signé en 1991 par l'État et les différentes collectivités locales: "Il ne peut que couvrir l'ensemble de la ville»" ${ }^{35}$. Le mot d'ordre de ce nouveau projet porte sur un renforcement de l'intercommunalité dans l'objectif de "faire de l'agglomération une vraie ville $»^{36}$.

En parenté avec le discours du Schéma directeur de 1974, Neptune, le projet urbain du centre, est défini comme une «extension du centre» et non pas un "morceau de ville», comparaison étant faite avec l'autre grande opération régionale Eurallile conduite parallèlement. En ce sens, Neptune œuvre pour l'agglomération: le centre ville devient centre d'agglomération en une véritable mutation, et même hypercentre de l'agglomération $^{37}$. L'agglomération se présente comme la solution au problème du morcellement de nouveau dénoncé: il est notamment proposé de ne pas «raisonner en zones». Le projet urbain vise à « redessiner une agglomération mieux structurée autour d'un centre, d'une première couronne, d'une seconde couronne». Il s'agit encore de «retisser l'ensemble urbain»».

De manière déterminée, le champ sémantique de la reprise (des espaces urbains) est investi. Les acteurs présentent la politique d'aménagement comme une requalification ou une reconquête. M. Lussault

35. Comprendre, penser, construire la ville, 1992, DAU, METT, p. 37.

36. Contrat d'agglomération, op. cit., p. 22.

37. Agur, 1990, Aménagement urbain du centre ville de Dunkerque - note d'orientation, p. 2-3. 
observe des termes tout à fait comparables pour Tours, sans les référer à une catégorie sémantique spécifique. Toutefois, le discours du maire J. Royer, développé à partir de 1977 dans son livre La cité retrouvée rend compte d'un positionnement plus temporel et d'un découpage différent des phases d'aménagement. Aucune référence à la reconstruction d'après-guerre ici, mais les années 90 sont entendues comme un renouveau tendant vers la Renaissance de la ville dont «l'urbanisme est un outil $»^{38}$. À Dunkerque, dès 1990, c'est un registre plus spatialisant qui est choisi. La dénomination usuelle de «requalification des espaces publics centraux» est acquise. Cette appellation induit des questionnements. De quel ordre est cette répétition? Que s'agit-il de reprendre ici?

Intervenant sur une ville déjà elle-même re-construite, l'aménagement des espaces publics réfère immédiatement à une historicité, quoique le terme n'en explicite pas le référent. Si celui-ci apparait comme devant être logiquement celui de la reconstruction dans la partie centrale, il devient plus problématique dans les autres parties de l'agglomération, la zone portuaire par exemple étant elle-même le résultat de plusieurs couches historiques superposées. La posture mérite donc d'être soulignée autant que son contenu: l'aménagement porte sur une ville aménagée; on parle d'aménagement de l'aménagé.

Les termes de reprise et de requalification rendent compte globalement de la prise de conscience d'un héritage constitué par l'aménagé. Tandis que le «système de centralités» instauré durant les décennies 60 et 70 «a produit des quartiers enclavés socialement», le projet urbain entend au contraire «prendre la ville comme un tout» en reliant centre ville et périphéries $^{39}$. La situation d'aménagement de l'aménagé donne lieu à une réflexion particulière sur le passé: la ville devient un appareil de vision particulièrement sensible des temporalités sociales et culturelles.

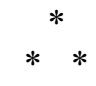

Les catégories du tout et de la partie participent de la reprise discontinue de la ville par les aménageurs. Elles traduisent la nécessité et tout autant les difficultés à donner nom à l'unité, c'est-à-dire à l'objet sur

38. M. Lussault, 1993, Tours: images de la ville et politique urbaine, Maison des sciences de la ville, Tours, p. 253.

39. Agur, 1990, Desserte du centre de Dunkerque par l'ouest, n. p. 
lequel portent les grandes interventions. L'urbanisme se révèle fortement réactif à son histoire, chaque phase se définissant par rapport aux précédentes, comme le montrent les champs sémantiques largement développés de la reprise et de la nouveauté. Ce vocabulaire témoigne dès lors de la construction par les acteurs du contenu de ce que Reinhart Koselleck a proposé comme deux catégories historiques fondamentales: le champ d'expérience et l'horizon d'attente ${ }^{40}$. La position des acteurs tient en effet dans la tension entre un répertoire d'expériences signifiantes (savoirs et pratiques) dont ils pourraient s'instruire et des formes de futurs possibles à inventer par le projet. Nous proposons de donner aux mots une dimension essentielle dans ces processus: les mots alimentent répertoires et projets. Autrement dit, nommer la ville participe à sa fabrique. D'ailleurs, ne pourrait-on pas distinguer mots à durée de vie courte $(Z U P, Z A C$ ou reconstruction) et mots à durée de vie plus longue (ville, agglomération, quartier, zone), les contenus de sens de ces derniers variant fortement au cours des années, mots qui viennent, partent et réapparaissent, que l'on pourrait appeler des mots «en écharpe» de l'urbain?

40. R. Koselleck, 1990, Le futur passé, Contribution à la sémantique des temps historiques, Paris, EHESS, 334 p. 